\title{
Reconstruction of fetal and infant anatomy using rapid prototyping of post-mortem MR images
}

\author{
Silvia Schievano $•$ Neil J. Sebire • Nicola J. Robertson • \\ Andrew M. Taylor $\cdot$ Sudhin Thayyil
}

Received: 19 April 2010 /Revised: 12 May 2010 / Accepted: 1 June 2010 / Published online: 19 June 2010

(C) European Society of Radiology 2010

\begin{abstract}
Objectives The recent decline in autopsy rates and lack of human anatomical material donated for research and training has resulted in issues for medical training in the United Kingdom. This study aims to examine the feasibility of making accurate three-dimensional (3D) models of the human body and visceral organs using post-mortem magnetic resonance (MR) imaging and rapid prototyping. Methods We performed post-mortem MR imaging using a 3D T2-weighted sequence in 11 fetuses and infants, before autopsy, using either a $1.5-\mathrm{T}$ or $9.4-\mathrm{T}$ MR scanner. Internal organs were reconstructed in silico and $3 \mathrm{D}$ models were created by rapid prototyping.

Results The median gestation of fetuses was 20 (range 1930 ) weeks and the median age of infants was 12 (range 816) weeks. Models created by rapid prototyping accurately depicted structural abnormalities and allowed clear visualisation of 3D relationships.
\end{abstract}

S. Schievano $\cdot$ A. M. Taylor $\cdot$ S. Thayyil

Cardiovascular Unit, UCL Institute of Child Health \& Great

Ormond Street Hospital for Children,

London, UK

N. J. Sebire

Histopathology, Great Ormond Street Hospital NHS Trust,

London, UK

N. J. Robertson

EGA, UCL Institute for Women's Health,

86-96 Chinies Mews,

WC1E 6HX London, UK

A. M. Taylor $(\square)$

Cardio-respiratory Unit, Great Ormond Street Hospital,

Great Ormond Street,

WC1N 3JH London, UK

e-mail: a.taylor@ich.ucl.ac.uk
Conclusions Accurate 3D modelling of anatomical features from post-mortem imaging in fetuses and infants is feasible. These models could have a large number of medical applications, including improved parental counselling, invaluable teaching resources and significant medico-legal applications to demonstrate disease or injury, without the need to show actual autopsy photographs.

Keywords Post-mortem imaging · Rapid prototyping · Magnetic resonance imaging . Computed tomography . Paediatrics

\section{Introduction}

Learning anatomy and pathology from the dissection of human bodies and examination of internal organs has traditionally played an important role in the training of doctors, particularly surgeons and pathologists; hence the human body is often referred to as the best medical textbook. However, organ retention issues and the subsequent introduction of the Human Tissue Act have led to a substantial decline in autopsy rates and therefore opportunities for research and training in the past decade in the United Kingdom [1, 2]. Consented autopsies in children have become virtually non-existent. Even when autopsies are performed, parents usually request that all internal organs be returned to the body for burial [1]. The Royal Colleges and the Department of Health have recently warned that shortage of donor bodies is putting medical teaching in the United Kingdom at risk [3].

In order to ensure that the important information provided by post-mortem assessment is not lost, there has been increasing interest from the medical profession, policy makers and the public to develop less invasive methods, 
such as magnetic resonance (MR) imaging and minimally invasive biopsies, to provide alternatives to conventional autopsy. Importantly, such approaches may be more acceptable to parents, as they do not involve open dissection of the body [4-7]. Large prospective studies are now ongoing in the United Kingdom, comparing the accuracy of such approaches to conventional autopsy. As part of one such study, we have been acquiring threedimensional (3D) MR data sets [7, 8] that can be reconstructed in 3D. Furthermore, we have used a technique known as rapid prototyping (3D printing) to build real 3D models of the right side of the heart and pulmonary trunk $[9,10]$, and have demonstrated that these models can improve clinical decision-making when treating patients. In the current study, we examine the feasibility and potential applicability of combining 3D post-mortem MR data from fetuses and infants with rapid prototyping to create models of post-mortem features, with the aim of improving understanding and teaching of pathological conditions. In addition, such models could be clinically useful and highly acceptable for explaining post-mortem findings to parents and demonstrating pathological findings in the medicolegal setting.

\section{Materials and methods}

We acquired post-mortem MR images in 11 fetuses and infants, prior to autopsy, using a 1.5-T MR scanner [Avanto, Siemens Medical Solutions, Erlangen, Germany $(n=10)$ ] or a 9.4-T MR scanner [VNMRS, Varian, Palo Alto, Calif., USA $(n=1)]$ for fetuses less than 20 weeks. These cases were selected from a post-mortem MR imaging database, to reflect common structural abnormalities and/or injuries that are encountered in routine clinical practice, and to demonstrate the use of the technique across a wide variety of organs. We used a T2-weighted 3D turbo spin echo sequence for MR imaging (Table 1). In one case, CT data of the skull was also acquired (SOMATOM Definition,
Siemens, Forchheim, Germany; parameters: collimation $2 \times$ $32 \times 0.6 \mathrm{~mm}$, rotation time $0.33 \mathrm{~s}$, pitch $0.2, \mathrm{kVp} 120$, mAs per rotation 375).

A biomedical engineer with 6 years' experience in image processing reconstructed digital 3D volumes of the body and internal organs using image post-processing software (Mimics 12.1, Materialise, Leuven, Belgium), as previously described [8-10]. In brief, the digital 3D datasets (.stl format) were imported into a rapid prototyping system where the volumes were converted into solid objects, layer by layer. A Z Corp printer (Z Corporation, Burlington, Mass., USA), that utilises inkjet print heads to deposit a binder into plaster powder was used for opaque and colour parts. An iPro SLA system (3D Systems Corporation, Rock Hill, S.C., USA) that employs an ultraviolet laser to cure liquid resin was used to build transparent models.

We have demonstrated excellent accuracy of the proposed methodology in a previous study, with operator error $<3.4 \%$ [9].

Ethics approval and role of funding agency

The local research ethics committee approved the study and parental consent for imaging was obtained as stipulated by the ethics committee for all consented post-mortems (04Q050841). For one case, MR imaging was performed as part of the request forensic assessment. The study sponsors had no role in the design or conduct of the study, in the collection, analysis, or interpretation of the data, or in the writing of the report. All the authors had access to the data and had the final responsibility in the decision to submit the paper for publication.

\section{Results}

Cases included eight fetuses [median gestation 20 (range 1630) weeks and weight $337(65-1,800) \mathrm{g}]$ and three infants [median age 12 (range 8-16) weeks and weight 14 (13.2-
Table 1 Typical MR imaging parameters for 3D (isotropic) T2-weighted fast spin echo (FSE) sequence

\begin{tabular}{lll}
\hline Parameter & 1.5 -T MR & $9.4-\mathrm{T} \mathrm{MR}$ \\
\hline Coil & Head (volume coil) & Rapid 39-, 72- or 150-mm volume coils \\
Effective TE $(\mathrm{ms})$ & 360 & 120 \\
TR $(\mathrm{ms})$ & 3,500 & 500 \\
Echo train length & 169 & 8 \\
Field of view (mm) & $125 \times 200 \times 64$ & $100 \times 50 \times 50$ \\
Matrix & $160 \times 252 \times 80$ & $512 \times 256 \times 256$ \\
Voxel dimensions $(\mathrm{mm})$ & $0.8 \times 0.8 \times 0.8$ & $0.2 \times 0.2 \times 0.2$ \\
Averages & 10 & 1 \\
Scan time & $\mathrm{Up} \mathrm{to} 50 \mathrm{~min}$ & $\mathrm{Up} \mathrm{to} 70 \mathrm{~min}$ \\
Flip angle & $90 / 120^{\circ}$ & $90 / 180^{\circ}$ \\
\hline
\end{tabular}


16.5) kg]. Post-mortem MR imaging was done at a median time of 4 (range 2-6) days after death. Three-dimensional reconstruction from the post-mortem MR images and rapid prototyping was feasible in all cases. Time for image elaboration was between 1 and $20 \mathrm{~h}$ and varied according to MR image quality and number of organs reconstructed. Rapid prototyping time was between 1 and $12 \mathrm{~h}$ according to volumes of the object, and costs were between $£ 10$ and $£ 240$, depending on the final model size. Structural anatomy and significant pathologies were easily identifiable by visual examination of the 3D models (Figs. 1, 2, 3 and 4).

\section{Discussion}

We have shown that accurate 3D models of fetal and infant anatomy, including internal organs, can be created by rapid prototyping of whole-body post-mortem MR images. Structural abnormalities of visceral organs and their relations to each other can be easily demonstrated, with a permanent record of the exact anatomical features for each individual case created. We feel that future application of such models may be useful in several ways:

- Improved understanding of complex congenital abnormalities

Congenital abnormalities often occur in small fetuses, where the exact relations may be difficult to appreciate because of the small size of internal organs and autolysis. Furthermore, whilst the pathologist performing the autopsy may delineate the features during dissection, demonstration of such findings to others at a later date, for counselling or teaching, has traditionally been difficult. Not only does rapid prototyping allow for a permanent 3D record of the features and relative position, but also simple magnification enables larger replicates of the pathological features (Fig. 1), making it easier to appreciate abnormal anatomy.

- Continued medical training from post-mortem data

Models of organs prepared by this technique may have important applications in medical training, particularly in anatomy and pathology. The rapid prototyping models can be stored long term without degradation or specific storage requirements. Such models can be annotated and since they are entirely non-identifiable, provide an ongoing unique teaching resource. For research purposes in the setting of rare congenital abnormalities this may also be important, since uncommon anomalies can be modelled, observed and categorised, without needing to retain either the fetus or organ, and precise replicas of rare findings could be produced multiple times and therefore shared across many training centres.

- Parental counselling

Clinicians may find these models particularly useful for explaining the abnormalities to parents, such as following a termination of pregnancy. Traditional post-mortem photographs are inappropriate for discussion with many parents, since the body is visible and blood will be present making the images unsuitable. However, this technique allows construction of a dissociated 'clean' model of the abnormal organ without requiring any autopsy photographs to be used. Some parents may indeed want to keep artificial replicas of their baby (Fig. 4) or organ, which could help in bereavement process.

- Demonstration of significant pathologies in medicolegal cases

In many medico-legal cases relating to the deaths of infants and children, juries and judges often depend heavily on evidence provided by pathologists, including post-mortem photographs and/or drawings, to under-
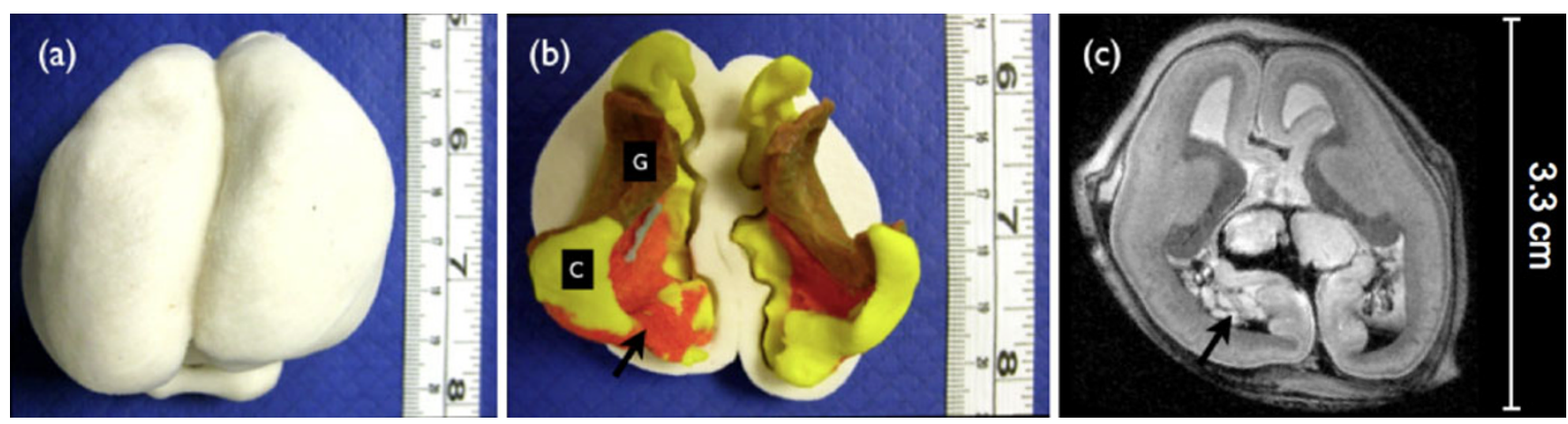

Fig. 1 Rapid prototyping of brain from high-field (9.4-T) MR images of a 16-week fetus. a External appearance of the brain. Note the smooth brain without sulci and gyri. b The brain cut open in the axial plane. $G$ germinal matrix, $C$ cerebrospinal fluid inside the lateral ventricles. Black arrows show the choroid plexus and bleeding into choroid plexus and ventricular cavity. c Corresponding 9.4-T MR images in the axial plane. The rapid prototyping model has been scaled to twice the original size. Conventional autopsy was difficult in this case due to autolysis of the brain and dimensions 
Fig. 2 a External rapid prototyping of a 30 -week fetus with sacrococcygeal teratoma. b Posterior and $\mathbf{d}$ anterior view after opening the body model. c Post-mortem MR image in the coronal plane. Teratoma is occupying most of the abdomen, displacing the intestines and other visceral organs. $H$ heart, $S$ spleen, $A$ adrenals, $I$ intestine, $L K$ left kidney, $R K$ right kidney, $L$ liver
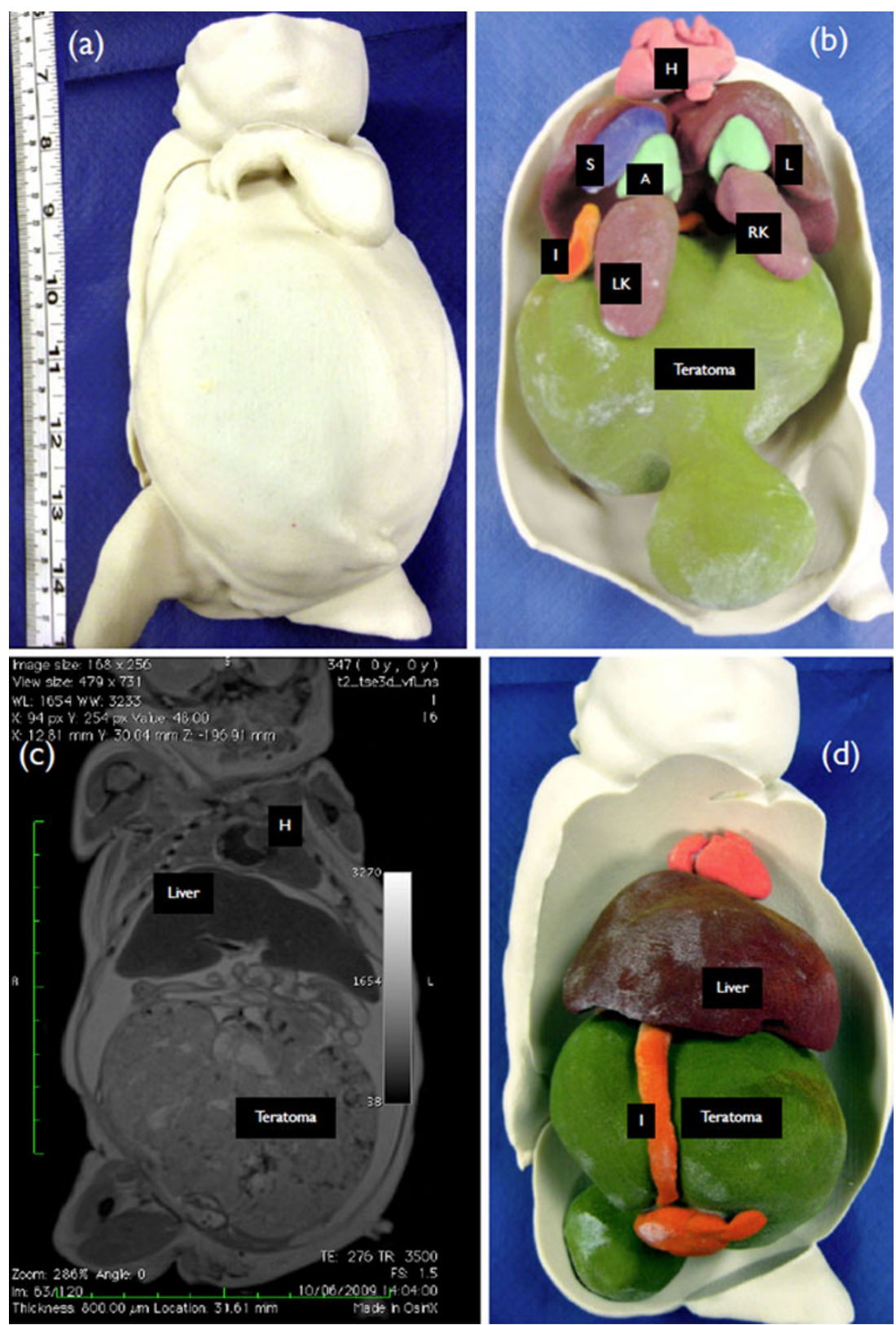

stand the nature and mode of injury. However, in many cases, for the reasons stated above, autopsy photographs are deemed inappropriate for use in court due to the presence of blood or other non-specific post-mortem features. Rapid prototyping models allow demonstration of only the significant pathological findings with clear annotations and may be useful in demonstrating the mode of injury in such cases in the context of legal evidence (Fig. 3). Reconstruction of the anatomical features may also be valuable in other settings, such as mass disasters or cases in which a body is decomposed, so that conventional autopsy is difficult.

- Use of rapid prototyping for in-utero fetal diagnosis and management
With development of rapid multi-slice snapshot MR sequences, 3D volume reconstruction of fetal organs has become possible [11]. Therefore, rapid prototyping may have significant implications for in-utero fetal MR imaging, particularly in understanding the exact relations of the internal organs in cases of complex anomalies such as fetal diaphragmatic hernias and conjoined twins [12], which may allow planning of subsequent surgical management in such fetuses in the future. In addition, facial models created by rapid prototyping of in-utero MR, may be useful in identifying facial dysmorphism in cases of genetic syndromes and allow clear demonstration of the extent of facial clefting. Modelling of the skeleton would be useful for diagnosing cases of skeletal dysplasia. 
Fig. 3 a-c Fractured skull and underling parenchymal bleed in the brain of an infant. d

Corresponding post-mortem MR image in the sagittal plane. Black arrows indicate the bleed. These findings were confirmed at autopsy. This model was built by combining and registering CT images, which provided the 3D skull structure, with MR data that were used to reconstruct the bleed volumes and positions
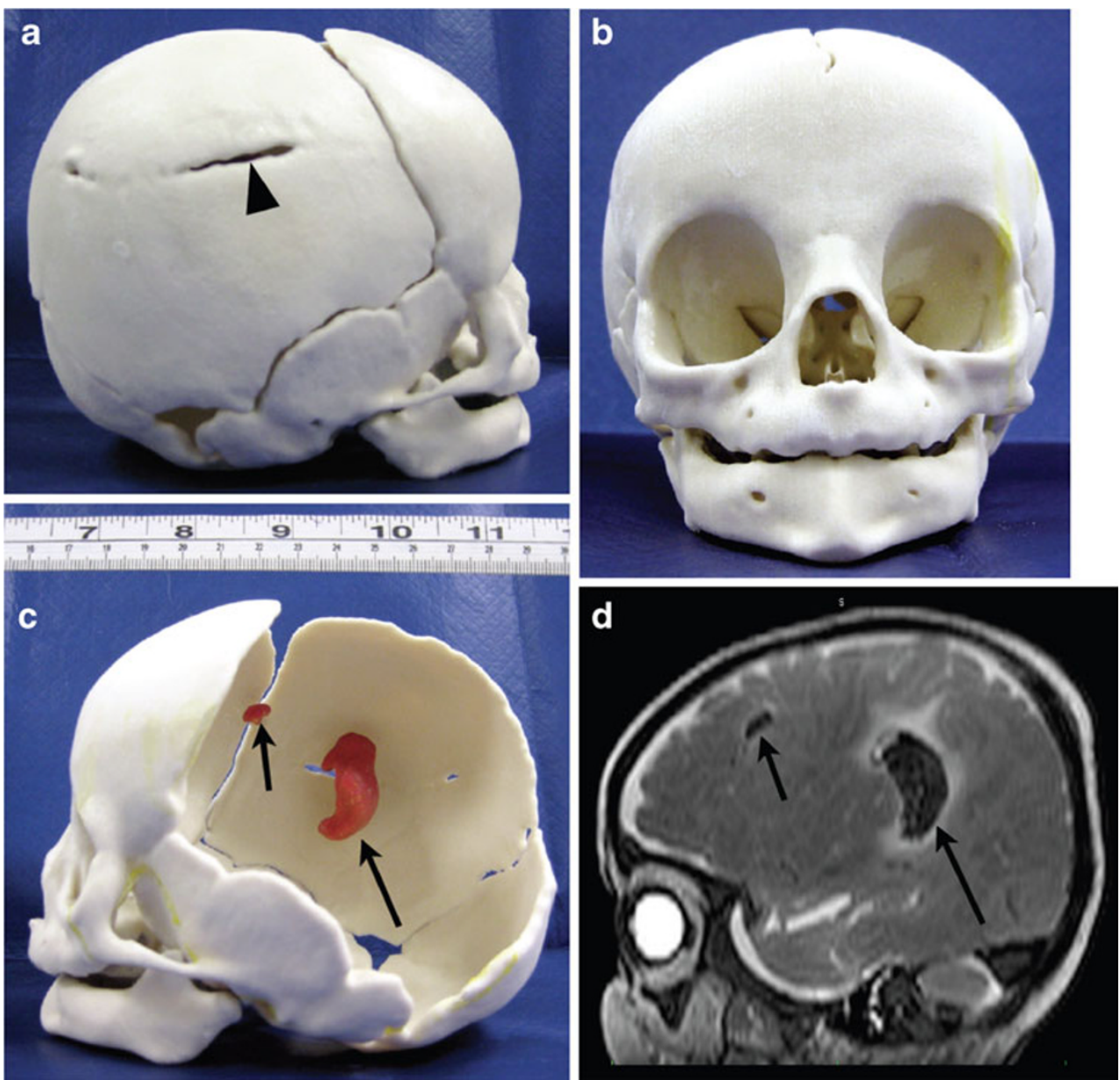

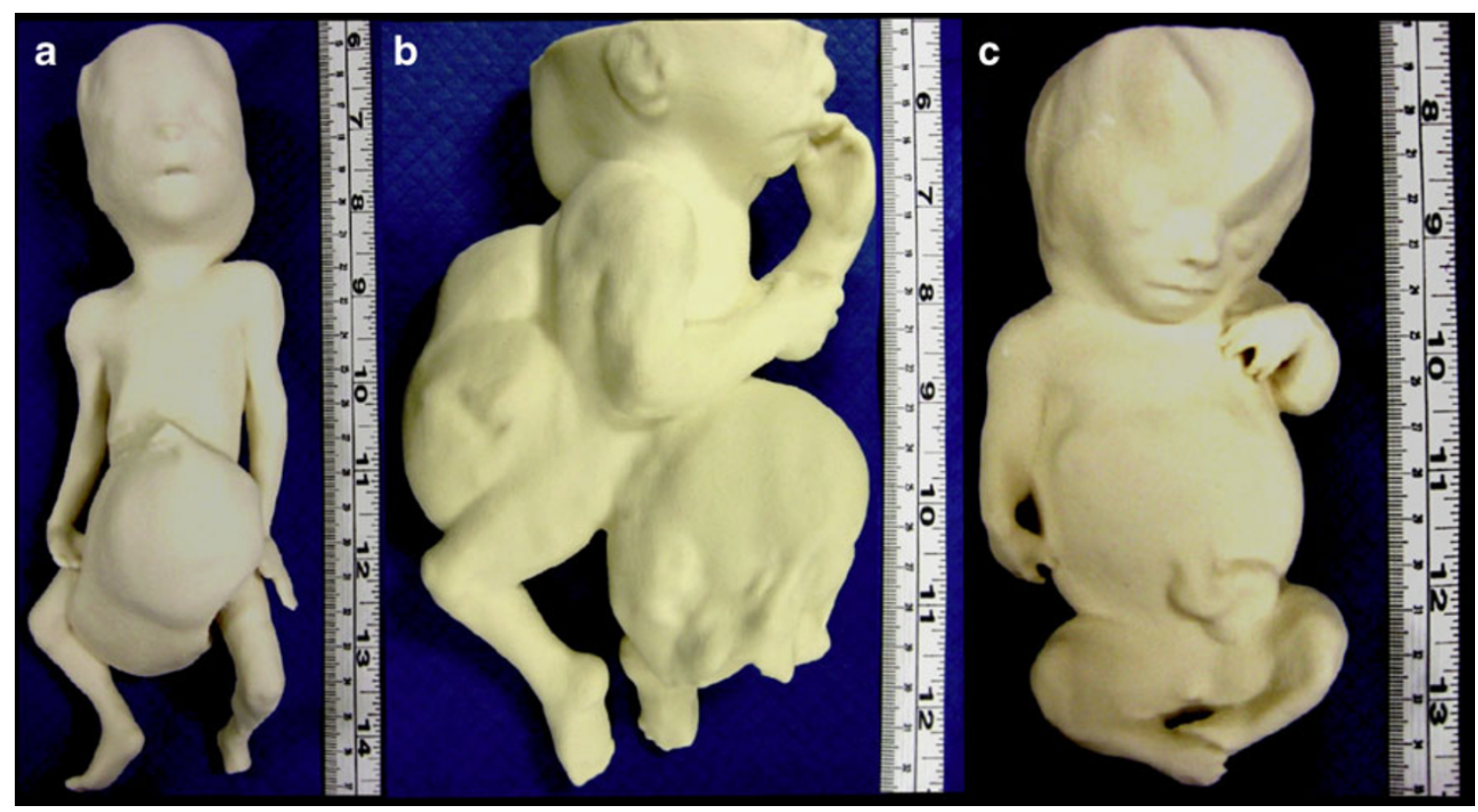

Fig. 4 External body rapid prototyping of (a) a 19-week fetus with a large exompholos, (b) an 18-week fetus terminated for a large myelemenigocele and exompholos (pentalogy of Cantrell) and (c) a 20-week fetus with short limb skeletal dysplasia 
- A replacement for plastination

Over recent years, there has been controversy and public outcry related to the use of plastination to exhibit the human body and aid in medical training [13]. For the process of plastination, body parts are immersed in acetone chilled to $13^{\circ} \mathrm{F}$, and the water removed from every cell. The water is then replaced with a molten plastic material that later hardens. The parts retain their colour and shape, and many organs have a plastic appearance [13]. However, plastination requires the use of human bodies and therefore raises issues of acceptability; the rapid prototyping technique we describe does not involve use of any human tissue.

Many of these described applications for use of rapid prototyping methods are speculative. Ultimately, the utility of these applications will need to be explored with future studies.

At present, preparation of rapid prototyping models require specialist skills, expertise and equipment, which is likely to be available only in few selected centres in the United Kingdom. However, it would be possible to perform post-mortem MR imaging in most large hospitals and these images could be transmitted easily to a specialist centre for subsequent analysis and preparation of the models. Furthermore, with continued refinement of the rapid prototyping technique it will be possible to create future models that have the similar consistency, feel and colour to native human organs.

In summary, post-mortem MR imaging and rapid prototyping can create accurate $3 \mathrm{D}$ models of fetal and infant anatomical features and these models may have many potentially important medical and medicolegal applications, whilst removing the need for retention of organs.

Acknowledgements The authors would like to thank Dr. Matteo Bonacina from ProMEV SRL, Lecco, Italy for his technical support. This study was supported by funding from the Royal Academy of Engineering/EPSRC, the Higher Education Funding Council for England, the UK National Institute of Health Research, the UK Department of Health and Comprehensive Biomedical Research Centre and the Fondation Leducq.

\section{References}

1. Royal College of Paediatrics and Child Health (2002) The future of paediatric pathology services. www.rcpch.ac.uk/doc.aspx? id Resource $=1742$

2. Report of a census of organs and tissues retained by pathology services in England. Conducted in 2000 by the Chief Medical Officer. www.dh.gov.uk/en/Publicationsandstatistics/Publications/ PublicationsStatistics/DH_4006720

3. Shortage of human cadavers for medical training. http://www.biomedicine.org/medicine-news/Shortage-of-Human-Cadavers-forMedical-Training-19041-1/

4. Brookes JA, Hall-Craggs MA, Sams VR, Lees WRC (1996) Non-invasive perinatal necropsy by magnetic resonance imaging. Lancet 348:1139-1141

5. Cohen M, Whitby EH (2007) The use of magnetic resonance in the hospital and coronial pediatric postmortem examination. Forensic Sci Med 3:289-296

6. Griffiths PD, Paley MN, Whitby EH (2005) Post-mortem MRI as an adjunct to fetal or neonatal autopsy. Lancet 365:1271-1273

7. Thayyil S, Cleary JO, Sebire NJ, Scott RJ, Chong K, Gunny R, Owens CM, Olsen OE, Offiah AC, Parks HG, Chitty LS, Price AN, Yousry TA, Robertson NJ, Lythgoe MF, Taylor AM (2009) Post-mortem examination of human fetuses: a comparison of whole-body high-field MRI at $9.4 \mathrm{~T}$ with conventional MRI and invasive autopsy. Lancet 374:467-475

8. Thayyil S, Schievano S, Robertson NJ, Jones R, Chitty LS, Sebire NJ, Taylor AM (2009) A semi-automated method for non-invasive internal organ weight estimation by post-mortem magnetic resonance imaging in fetuses, newborns and children. Eur $\mathrm{J}$ Radiol 72(2):321-326

9. Schievano S, Migliavacca F, Coats L, Khambadkone S, Carminati M, Wilson N, Deanfield JE, Bonhoeffer P, Taylor AM (2007) Percutaneous pulmonary valve implantation based on rapid prototyping of right ventricular outflow tract and pulmonary trunk from MR data. Radiology 242:490-497

10. Armillotta A, Bonhoeffer P, Dubini G, Ferragina S, Migliavacca F, Sala G, Schievano S (2007) Use of rapid prototyping models in the planning of percutaneous pulmonary valved stent implantation. Proc Inst Mech Eng H 221(4):407-416

11. Hayat T, Jiang S, Allsop J, Hajnal J, Rutherford M (2008) Quantification of human fetal brain development in utero using multislice snapshot MRI with 3D volume reconstruction. Ultrasound Obstet Gynecol 31:597-609

12. Christensen AM, Humphries SM, Goh KYC, Swift D (2004) Advanced "tactile" medical imaging for separation surgeries of conjoined twins. Childs Nerv Syst 20:547-553

13. Body display 'may threaten donations'. http://news.bbc.co.uk/1/ hi/england/2038126.stm 